\title{
The new basal insulin analog glargine U-300 enables flexible injection schedule
}

\author{
Uru Nezu Osada ${ }^{1}$, Yasuo Terauchi $^{2}$ \\ ${ }^{1}$ Department of Diabetes and Endocrinology, Saiseikai Yokohamashi Nanbu Hospital, 3-2-9 Konandai, Konan-ku, Yokohama, Japan; ${ }^{2}$ Department of \\ Endocrinology and Metabolism, Graduate School of Medicine, Yokohama City University, 3-9 Fukuura, Kanazawa-ku, Yokohama, Japan \\ Correspondence to: Yasuo Terauchi, MD, PhD. Department of Endocrinology and Metabolism, Graduate School of Medicine, Yokohama City \\ University, 3-9 Fukuura, Kanazawa-ku, Yokohama 236-0004, Japan. Email: terauchi-tky@umin.ac.jp. \\ Provenance: This is an invited Editorial commissioned by the Section Editor Kaiping Zhang (AME College, AME Group, China) and the Guest \\ Section Editor Hengrui Liang (Nanshan Clinical Medicine School, Guangzhou Medical University, Guangzhou, China). \\ Comment on: Home PD, Bergenstal RM, Bolli GB, et al. Glycaemic control and hypoglycaemia during 12 months of randomized treatment with \\ insulin glargine $300 \mathrm{U} / \mathrm{mL}$ versus glargine $100 \mathrm{U} / \mathrm{mL}$ in people with type 1 diabetes (EDITION 4). Diabetes Obes Metab 2018;20:121-8.
}

Submitted Aug 14, 2018. Accepted for publication Aug 18, 2018.

doi: 10.21037/atm.2018.08.25

View this article at: http://dx.doi.org/10.21037/atm.2018.08.25

Recently, Home et al. published a pre-planned continuation trial of EDITION 4 (1). EDITION 4 was a 6 -month long, multicenter, randomized, and open-label clinical trial involving patients with type 1 diabetes mellitus (T1DM) who received a morning or evening injection of either $300 \mathrm{U} / \mathrm{mL}$ of insulin glargine (Gla-300) or $100 \mathrm{U} / \mathrm{mL}$ of insulin glargine (Gla-100) (1) to evaluate the safety, tolerability, and efficacy of both the dosages of the basal insulin analog. After 12 months in the pre-planned continuation trial, the data showed that although Gla-300 and Gla-100 enabled equivalent glycemic control, there was a lower risk of nocturnal hypoglycemia with Gla-300 than with Gla-100. The trial also reported two other notable findings.

First, weight gain due to Gla-300 was lower than that due to Gla-100, even though the former's insulin content was higher than the latter's. This was not in accordance with our understanding, which is that higher the insulin, higher is the weight gain, because the glucose-lowering effect of insulin is mediated by the glucose uptake of cells such as adipocytes. Indeed, DCCT and UKPDS have shown that intense insulin therapy leads to considerable weight gain in patients with T1DM as well as type 2 diabetes $(2,3)$. Second, the morning or evening injection of Gla-300 did not produce differing glucose profiles or hypoglycemia frequency (1), suggesting that the Gla-300 injection schedule can be flexible.

These findings may be attributable to the pharmacokinetic/ pharmacodynamic (PK/PD) of Gla-300 and the insulin titration algorithm. In fact, several euglycemic clamp studies on Gla-300 and Gla-100 in patients with T1DM have found that Gla-300 shows a flatter PK/PD profile over $24 \mathrm{~h}$ and a more prolonged activity of up to $36 \mathrm{~h}$ than Gla-100 (4). Furthermore, continuous glucose monitoring (CGM) analyses showed that the 24-h glucose profile of Gla-300 was flatter than that of Gla-100 (5). Collectively, these findings suggest that Gla-300 reduces the risk of hypoglycemia.

Gla-300 and Gla-100 did not show differing hypoglycemia frequencies in EDITION 4 (1). However, in EDITION JP1, which was a randomized controlled trial involving Japanese patients with T1DM, Gla-300 showed a lower hypoglycemia frequency during both daytime and nighttime than Gla-100 (6). Also in trials with Japanese type 2 diabetes, which were EDITION JP2 (7) and its extension study (8), annualised rates of hypoglycemia was 30\% less in Gla-300 compared to Gla-100. Although the reason for this variation is unclear, it should be noted that mild hypoglycemia is undetectable by SMPG. We speculate that the high glucose fluctuation induced by Gla-100 may increase appetite for snacks or reduce physical activity. Such lifestyle changes may have obscured overt hypoglycemia in EDITION 4.

A CGM study examined the 24-h glucose profiles of Gla-100 and Gla-300 with different injection timings (5). 
Although the glucose profiles of Gla-300 morning and evening were nearly superimposable, Gla-100 showed more excursion in glucose levels from 2 to 8 am in the Gla100 morning injection group. This is likely a result of the basal insulin waning of Gla-100 toward the end of the 24-h dosing interval, which is not masked by the use of rapidacting insulin during this early morning period. Basal insulin is commonly titrated based on fasting blood-glucose levels, thereby causing a more aggressive up-titration in morning injection than in evening injection. In fact, EDITION 4 (1) results showed that the percentage of basal insulin was the highest for the morning injection of Gla-100 (59.5\%; percentage of basal insulin for the evening injection, $51.7 \%$ ), whereas Gla-300 was equally up-titrated between the two injection schedules (morning vs. evening; 58.2\% vs. 56.1\%).

Patients with T1DM require a flexible schedule for insulin injection depending on their lifestyle and preferences. Our survey on the expectations from diabetes care showed a perception gap between patients and healthcare providers (9). The survey was conducted between March 25, 2016 and April, 18,2016 via a web-based questionnaire. The participants were 712 patients with diabetes aged 20 years and older, who were being treated with basal insulin (81 patients with T1DM and 631 patients with T2DM); 221 physicians treating patients with diabetes with basal insulin; and 110 nurses working on insulin therapy in departments of internal medicine or specialized departments of diabetes/endocrinology. In total, $30.2 \%$ patients had satisfactory glycemic control; of them, $37.2 \%$ had experienced hypoglycemia within the previous 3 months. Moreover, only 55\% patients were satisfied with their glycemic control, which was lower than that estimated by the physicians $(69 \%)$. A total of $93.2 \%$ patients desired an improvement in their glycemic control, which was higher than that estimated by the physicians (73.3\%) and nurses (64.9\%). Approximately $50 \%$ of the patients were worried about their condition: $72.5 \%$ were worried about complications, $48.5 \%$ about hypoglycemia, and $43.0 \%$ about glycemic variability. These results suggest that the physicians and nurses underestimate patient interest in diabetes management and overestimate treatment satisfaction (9). The patients were more concerned about the risk of hypoglycemia, wanted more stable glycemic control, and desired more information on new treatment methods and basal insulin formulations than that expected by the physicians. Healthcare providers may be able to improve diabetes care by better understanding management expectations of the patients and sharing them with other team members. In this context, Gla-300 may reduce the burden of insulin therapy and improve patient satisfaction by enabling a flexible injection schedule.

Insulin degludec $100 \mathrm{U} / \mathrm{mL}$ (IDeg-100) is another long-acting basal insulin analog enabling flexible injection schedule (10). Although a detailed comparison between the benefits of Gla-300 and the benefits of IDeg is limited, a recent euglycemic clamp study by Bailey et al. reported that Gla-300 shows a more stable PK/PD profile than insulin IDeg-100 (11). More recently, at the $78^{\text {th }}$ American Diabetes Association Scientific Sessions held at Orland, USA, in June 2018, several studies comparing the efficacy of Gla300 vs. Deg-100 were presented. BRIGHT study was the first head-to-head randomized controlled trial of Gla-300 and IDeg-100 in patients with insulin-naive type 2 diabetes. Glycemic control was similar between Gla-300 and IDeg100. The rate of hypoglycemia was relatively lower with Gla-300, by $14 \%$ at any time of day and $19 \%$ at night, despite higher final daily doses of Gla-300 vs. IDeg-100 (12). The reduction of hypoglycemia was prominent during the first 3 months after Gla-300 initiation (23\% less) (13). These reports imply the superiority of Gla-300; however, the opposite insight was also reported. In CONFIRM study, which was a propensity-score matched observational study, glycemic control and rate of hypoglycemia were both favorable in IDeg-100. In addition, the rate of treatment discontinuation was $37 \%$ higher with Gla-300 (14).

Overall, Gla-300 is likely to have superior clinical efficacy than conventional Gla-100, in context of the stability of glycemic control and elasticity of the injection schedule. The superiority of Gla-300 to IDeg-100 is still controversial and needs to be elucidated with further studies.

\section{Acknowledgments}

None.

\section{Footnote}

Conflicts of Interest: UN. Osada has received honoraria for speakers' bureau from Amgen Astellas BioPharma, Daiichi Sankyo, Eli Lilly Japan K.K., Kowa, Kyowa Hakko Kirin, Medtronic, Mitsubishi Tanabe Pharma, Nippon Boehringer Ingelheim, Novartis Pharma, Novo Nordisk, Sanofi K.K., and Sumitomo Dainippon Pharma. Y Terauchi has received honoraria for speakers' bureau from Astellas Pharma, AstraZeneca K.K., Bayer Yakuhin, Daiichi Sankyo, Sumitomo Dainippon Pharma, Eli Lilly Japan K.K., Kowa, MSD K.K., Mitsubishi Tanabe Pharma, Nippon Boehringer Ingelheim, Novo Nordisk, Ono Pharmaceutical, 
Sanwa Kagaku Kenkyusho, Sanofi K.K., Shionogi \& Company, Taisho Toyama Pharmaceutical, and Takeda Pharmaceutical Company along with grants from Astellas Pharma, AstraZeneca K.K., Bayer Yakuhin, Daiichi Sankyo, Sumitomo Dainippon Pharma, Eli Lilly Japan K.K., Kowa, MSD K.K., Mitsubishi Tanabe Pharma, Nippon Boehringer Ingelheim, Novo Nordisk, Ono Pharmaceutical, Pfizer Japan, Sanwa Kagaku Kenkyusho, Sanofi K.K., Shionogi \& Company, and Takeda Pharmaceutical Company. Y Terauchi was also a principal investigator in a Japanese phase IIIa trial of insulin glargine U-300 (EDITION JP2).

\section{References}

1. Goldman J, White JR. New Insulin Glargine $300 \mathrm{U} / \mathrm{mL}$ for the Treatment of Type 1 and Type 2 Diabetes Mellitus. Ann Pharmacother 2015;49:1153-61.

2. Nathan DM, Genuth S, Lachin J, et al. The effect of intensive treatment of diabetes on the development and progression of long-term complications in insulindependent diabetes mellitus. N Engl J Med 1993;329:977-86.

3. Intensive blood-glucose control with sulphonylureas or insulin compared with conventional treatment and risk of complications in patients with type 2 diabetes (UKPDS 33). UK Prospective Diabetes Study (UKPDS) Group. Lancet 1998;352:837-53.

4. Becker RH, Dahmen R, Bergmann K, et al. New insulin glargine 300 Units $\cdot \mathrm{mL}-1$ provides a more even activity profile and prolonged glycemic control at steady state compared with insulin glargine 100 Units · mL-1. Diabetes Care 2015;38:637-43.

5. Bergenstal RM, Bailey TS, Rodbard D, et al. Comparison of Insulin Glargine 300 Units $/ \mathrm{mL}$ and 100 Units $/ \mathrm{mL}$ in Adults With Type 1 Diabetes: Continuous Glucose Monitoring Profiles and Variability Using Morning or Evening Injections. Diabetes Care 2017;40:554-60.

6. Matsuhisa M, Koyama M, Cheng X, et al. New insulin glargine $300 \mathrm{U} / \mathrm{ml}$ versus glargine $100 \mathrm{U} / \mathrm{ml}$ in Japanese adults with type 1 diabetes using basal and mealtime insulin: glucose control and hypoglycaemia in a randomized controlled trial (EDITION JP 1). Diabetes Obes Metab 2016;18:375-83.

7. Terauchi Y, Koyama M, Cheng X, et al. New insulin glargine $300 \mathrm{U} / \mathrm{ml}$ versus glargine $100 \mathrm{U} / \mathrm{ml}$ in Japanese people with type 2 diabetes using basal insulin and oral antihyperglycaemic drugs: glucose control and hypoglycaemia in a randomized controlled trial (EDITION JP 2). Diabetes Obes Metab 2016;18:366-74.
8. Terauchi Y, Koyama M, Cheng X, et al. Glycaemic control and hypoglycaemia with insulin glargine 300 $\mathrm{U} / \mathrm{mL}$ compared with glargine $100 \mathrm{U} / \mathrm{mL}$ in Japanese adults with type 2 diabetes using basal insulin plus oral anti-hyperglycaemic drugs (EDITION JP 2 randomised 12-month trial including 6-month extension). Diabetes Metab 2017;43:446-52.

9. Terauchi Y, Naito Y. Gap between patient expectations and health care provider perceptions. Therapeutic Research 2016;37:757.

10. Meneghini L, Atkin SL, Gough SC, et al. The efficacy and safety of insulin degludec given in variable oncedaily dosing intervals compared with insulin glargine and insulin degludec dosed at the same time daily: a 26-week, randomized, open-label, parallel-group, treat-to-target trial in individuals with type 2 diabetes. Diabetes Care 2013;36:858-64.

11. Bailey TS, Pettus J, Roussel R, et al. Morning administration of $0.4 \mathrm{U} / \mathrm{kg} /$ day insulin glargine $300 \mathrm{U} / \mathrm{mL}$ provides less fluctuating 24-hour pharmacodynamics and more even pharmacokinetic profiles compared with insulin degludec $100 \mathrm{U} / \mathrm{mL}$ in type 1 diabetes. Diabetes Metab 2018;44:15-21.

12. Cheng AY, Rosenstock J, Ritzel R, et al. Similar Glycemic Control and Less or Comparable Hypoglycemia with Insulin Glargine $300 \mathrm{U} / \mathrm{mL}$ (Gla-300) vs. Degludec $100 \mathrm{U} / \mathrm{mL}$ (IDeg-100) in Insulin-Naïve T2DM on Antihyperglycemic Drugs \pm GLP-1 RAs-The BRIGHT Randomized Study. Diabetes 2018;67.

13. Bolli GB, Cheng A, Bosnyak Z, et al. Lower Hypoglycemia Rates with Insulin Glargine $300 \mathrm{U} / \mathrm{mL}$ (Gla-300) vs. Insulin Degludec 100 U/mL (IDeg-100) in Insulin-Naïve Adults with T2DM on Oral Antihyperglycemic Therapy \pm GLP-1RA-The BRIGHT Randomized Study. Diabetes 2018;67.

14. Tildi J, Haldrup S, Sandberg V, et al. Clinical Outcome Assessment of the Effectiveness of Insulin Degludec (Degludec) in Real-life Medical Practice (CONFIRM)—A Comparative Effectiveness Study of Degludec and Insulin Glargine 300U/mL (Glargine U300) in Insulin-Naïve Patients with Type 2 Diabetes (T2D). Diabetes 2018;67.

Cite this article as: Osada UN, Terauchi Y. The new basal insulin analog glargine U-300 enables flexible injection schedule. Ann Transl Med 2018;6(Suppl 1):S1. doi: 10.21037/ atm.2018.08.25 